\title{
Embodied and situated language processing
}

The sixth annual conference on embodied and situated language processing (ESLP) will take place in Potsdam/ Germany from 29 to 31 July 2013.

The meetings aim to bring together researchers working on the interaction of language and visual/motor processing in embodied, situated, and language-for-action research traditions. A further goal is to unite converging and complementary evidence from behavioral, neuroscientific, neuropsychological and computational methods.
The focus of ESLP 2013 is on the motor processes that occur before, during and after language comprehension, their function and time course.

Keynotes will be delivered by Anna Borghi, Barbara Kaup, Tatjana Nazir, Friedemann Pulvermüller, and Gabriella Vigliocco.

Further details can be found at www.eslp-conference.org/. 